\title{
Bifurcation picture and stability of the gap and out-gap discrete solitons
}

\author{
L. Kroon \\ Department of Physics, Chemistry and Biology, Linkoping University, Linkoping SE-581 83, Sweden \\ M.M. Bogdan and A.S. Kovalev \\ B. Verkin Institute for Low Temperature Physics and Engineering of the National Academy of Sciences of Ukraine \\ 47 Lenin Ave., Kharkov 61103, Ukraine \\ E-mail:kovalev@ilt.kharkov.ua \\ E.Yu. Malyuta \\ Electrophysical Scientific and Technical Center, 28 Chaikovskogo Str., Kharkov 61002, Ukraine
}

Received March 12, 2007

\begin{abstract}
The dynamics of a quaternary fragment of a discrete system of coupled nonlinear oscillators with modulated frequency parameters is investigated and the stability of its gap and out-gap soliton-like excitations is studied.
\end{abstract}

PACS: 05.45.-a Nonlinear dynamics and chaos;

63.10.+a General theory;

42.79. Gn Optical waveguides and couplers.

Keywords: linear gap, nonlinear gap, stability, modulated systems.

\section{Introduction}

A conception of the gap (Bragg) soliton has appeared for the first time in nonlinear optics $[1,2]$. These solitons can exist in nonlinear systems with spatial periodicity of some material parameters and possess the frequencies in the gap of the spectrum of linear excitations. The interest to the problem is caused by the fact that the group velocity of linear waves, linear pulses and solitons tends to zero at the boundary of the gap. The velocity of optical pulse is reduced significantly in material domains with modulated parameters which are inserted into the optical fiber, and this effect could be used in nonlinear optical devices. Solitons with the frequencies outside of the gap, the out-gap solitons, were first studied in [3]. This type of embedded solitons has frequencies inside the band of linear excitations and nonzero asymptotics at infinity. Therefore in contrast to the gap soliton the out-gap one has infinite norm. The gap- and out-gap solitons in discrete systems with alternating atom or spin characteristics, e.g. in diatomic lattices and two-sublattice magnets, were investi- gated by many authors. A lot of obtained results were similar to those for extended systems, while some finite-size models were solved exactly in special cases. However two important questions remain still open: (i) how the gap soliton transforms into the out-gap one at the boundary of the frequency gap of linear waves («linear gap»), and (ii) what are stability properties of the gap and out-gap solitons.

It is well-known [4] that many aspects of soliton dynamics of nonlinear systems can be elucidated in the framework of models with a finite number of degrees of freedom. The simplest discrete modulated system which permits of an existence of analogues of the gap and out-gap excitations represents the ring of four coupled nonlinear oscillators with alternating frequency parameters [5]. To date this model is of a great interest for low-temperature physics due to a topical problem of magnetic molecular nanoclusters [6]. It is known that in systems of finite size or with a finite number of degrees of freedom quasi-solitons appear in the bifurcation manner beginning from the moment when the energy or system 
parameters, in particular a depth of the frequency modulation $\gamma$ [5], exceed the threshold values. The scenario of the birth of the analogue of the gap soliton in the quaternary model contains two bifurcations: at $\gamma_{c}=1.707$, where some new excitations appear in «nonlinear gap» (see below) and at $\gamma_{*}=1.750$, where analogues of the gap and out-gap modes appear. This bifurcation pattern was qualitatively depicted in the inset of Fig. 3 in [5]. For the first time these two bifurcations were discovered numerically by L. Kroon [7] who informed the authors of [5] about his results before the publication [5]. The exact bifurcation picture represented numerical results of [7] is shown in Fig. 1. The main goal of this paper is to reveal details of the scenario of transformation of the gap soliton analogue into out-gap soliton one and to analyse a stability of these nonlinear excitations.

\section{The model}

Nonlinear dynamics of a ring consisting of four coupled anharmonic oscillators (or classical spins with numbers $n=1,2,3,4)$ with a periodic modulation of the frequency parameter is considered in the framework of discrete nonlinear Schrödinger equation (DNLSE) [5]:

$$
i \dot{\psi}_{n}-\omega_{0}^{(n)} \psi_{n}+\varepsilon\left(\psi_{n+1}+\psi_{n-1}\right)+\left|\psi_{n}\right|^{2} \psi_{n}=0 .
$$

The corresponding Hamiltonian of the model has the form

$$
H=\sum_{n}\left[\omega_{0}^{(n}\left|\psi_{n}\right|^{2}-\varepsilon\left(\psi_{n} \psi_{n-1}^{*}+\psi_{n}^{*} \psi_{n-1}\right)-\left|\psi_{n}\right|^{4} / 2\right]
$$

with canonical conjugated variables $\left\{i \psi_{n}\right\},\left\{\psi_{n}^{*}\right\}$ and $\omega_{0}^{(n)} \equiv \omega_{a}\left(\omega_{0}^{(n)} \equiv \omega_{b}\right)$ when index $n$ is an odd (even) number. In addition to the Hamiltonian (2) also the norm (excitations number), defined by $N=\sum_{n}\left|\psi_{n}\right|^{2}$, is a conserved quantity for (1). The ratio $\gamma \equiv \omega_{b} / \omega_{a}$ (we suppose for definiteness $\gamma>1$ ) reflects the depth of modulation. The transformation $\psi_{n} \rightarrow \sqrt{\varepsilon} \psi_{n}, \omega_{0}^{(n)} \rightarrow \varepsilon \omega_{0}^{(n)}$ is invoked in normalizing the coupling $\varepsilon$ to unity. We will discuss the stationary states of the form $\psi_{n}^{(\omega)}(t)=\varphi_{n} \times$ $\times \exp (-i \omega t)$ with real amplitudes $\varphi_{n}$. In the linear limit $\left(\psi_{n} \rightarrow 0\right)$ the spectrum of normal modes contains only 4 frequencies for inphase and antiphase oscillations with $\omega_{\min , \max }=\left[\left(\omega_{a}+\omega_{b}\right) \mp \sqrt{\left(\omega_{b}-\omega_{a}\right)^{2}+16}\right] / 2$, and for the gap boundaries solutions with $\omega=\omega_{a}$ and with $\omega=\omega_{b}$. In the limit of a long chain these frequencies do not change but the domains $\left(\omega_{\min }, \omega_{a}\right)$ and $\left(\omega_{b}, \omega_{\max }\right)$ transform into two bands of the spectrum with the gap $\left(\omega_{a}, \omega_{b}\right)$. That is why we will call in our simple model this domain as a linear gap. In nonlinear case the frequen-

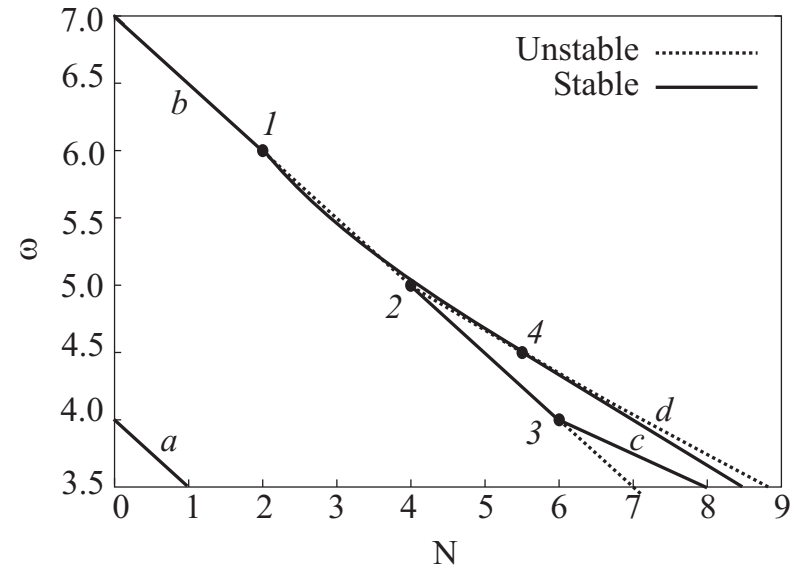

Fig. 1. Stationary solutions in the $(\omega, N)$ plane for $\gamma=1.75$. Solid (dotted) lines represent stable (unstable) regions of the solutions.

cies of aforesaid four «main nonlinear modes» decrease and «linear gap» transform into «nonlinear gap» with $\omega_{a, b}(N)=\omega_{a, b}-N_{a, b} / 2$ (see the lines $(a)$ and $(b)$ in Fig. 1 and Fig. 2). The frequencies $\omega_{a}$ and $\omega_{b}$ at the boundaries of the gap correspond to the antiphase oscillations $(\uparrow 0 \downarrow 0)$ and $(0 \Uparrow 0 \downarrow)$, respectively, where the zeros indicate immovable particles and the thickness of the arrows characterizes the relative amplitude of the oscillations. The frequencies for nonlinear oscillations depend not only on the parameter $\gamma$, but also on the amplitude and, hence, implicitly on the norm $N$ and the energy $E$ defined from the Hamiltonian (2). The «spectral» dependence $E=E(N)$ of the system is uniquely determined by the characteristic $\omega=\omega(N)$ due to the fulfillment of the relation $\omega=d E / d N$ for monochromatic oscillations. The most interesting for us are the upper boundary of the nonlinear gap (line $b$ in Fig. 1) and the analogue of the gap and out-gap modes (line $e$ in Fig. 2), bifurcating from the boundary.

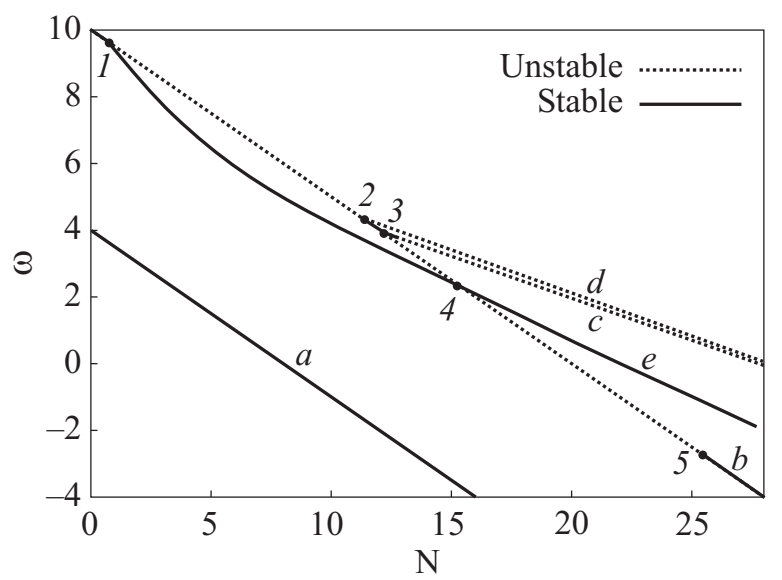

Fig. 2. Stationary solutions in the $(\omega, N)$ plane for $\gamma=2.5$, which is the threshold for the linear stability of the gap and out-gap solitons. 


\section{The stability problem}

The stability of the solution $\psi_{n}^{(\omega)}(t)$ is analyzed by adding the perturbation $\varphi_{n}(t)=\alpha_{n} \exp (-i \Omega t)+$ $+\beta_{n}^{*} \exp \left(i \Omega^{*} t\right)$ to its time-independent amplitude $\varphi_{n}$. Linearization of Eq. (1) around the stationary solution yields the eigenvalue problem

$$
\begin{gathered}
\left(\omega-\omega_{0}^{(n)}+2 \varphi_{n}^{2}\right) \alpha_{n}+\alpha_{n+1}+\alpha_{n-1}+\varphi_{n}^{2} \beta_{n}=-\Omega \beta_{n}, \\
\left(\omega-\omega_{0}^{(n)}+2 \varphi_{n}^{2}\right) \beta_{n}+\beta_{n+1}+\beta_{n-1}+\varphi_{n}^{2} \alpha_{n}=\Omega \beta_{n} .
\end{gathered}
$$

The linear stability of the stationary solution is equivalent to all eigenfrequencies $\Omega$ being real.

(i) The stability of $b$-mode. This problem can be solved analytically. Introducing the notations $A=\omega_{a}-\omega, B=$ $=\omega_{b}-\omega, \omega_{0}=\left(\omega_{a}+\omega_{b}\right) / 2$ and the width $\Delta \equiv B-A=$ $=(\gamma-1) \omega_{0}$ of the linear gap, the solution for the upper boundary of the «nonlinear» gap ( $b$-mode) can be written as $\left\{\varphi_{n}^{(b)}\right\}=\{0, \sqrt{B}, 0,-\sqrt{B}\}$ [5] and the eigenvalues $\Omega$ are found to satisfy the equation

$$
\Omega^{2}\left(\Omega^{2}-A^{2}\right)\left(\Omega^{4}-\left(A^{2}+8\right) \Omega^{2}+8 A B+16\right)=0 .
$$

The root $\Omega=0$ corresponds to «phase mode» and the $\operatorname{roots} \Omega= \pm A$ are real, whereas the remaining eigenvalues

$$
\Omega= \pm \sqrt{\left(A^{2}+8\right) / 2 \pm \sqrt{\left(A^{2}+8\right)^{2} / 4-8(A B+2)}}
$$

are complex whenever $A\left(A^{3}-16 A-32 \Delta\right)<0$. Denoting $\omega_{ \pm}=\sqrt[3]{16 \Delta \pm \sqrt{(16 \Delta)^{2}-(16 / 3)^{3}}}$ the criterion leads to the oscillatory instability [2] in the region $0<A<\omega_{+}+\omega_{-}$, which appears through Krein collisions [8] and manifests itself through resonances of the internal modes. One may note from Fig. 1 that there exists two windows of instability for $b$-mode. The first one is bounded by the bifurcation point 1 of gap $e$-mode and the bifurcation point 2 in Fig. 1. For large $\gamma$ this is the bifurcation point for unstable $d$-mode (Fig. 2). A second interval developing for $\Delta<4 /(3 \sqrt{3})$, is ruled out by the constraint $B=A+\Delta>0$ and is bounded by the point 3 for the bifurcation of $c$-mode $\left\{\varphi_{n}^{(c)}\right\}==\{\sqrt{A}, \sqrt{B},-\sqrt{A},-\sqrt{B}\}$ [5] at the frequency $\omega=\omega_{a}$ (the low boundary of the linear gap). If $A B+2<0$ is satisfied two of the eigenvalues (5) are not real. Applying the dependence $\omega_{b}(N)=\gamma \omega_{a}-N / 2$ of frequency on norm $N$, inequality gives an interval $\left|\omega_{b}(N)-\omega_{0}\right|<\sqrt{(\Delta / 2)^{2}-2}$, for which the solutions $\left\{\varphi_{n}^{(b)}\right\}$ is unstable, if and only if $\Delta>2 \sqrt{2} \equiv \Delta_{c}$. For large $\gamma$ (see Fig. 2) $c$-mode is stable only in the vicinity of point 3. The lower boundary of nonlinear gap corresponds to $a$-mode which is linearly stable for all values of parameter $\gamma$. (ii) The stability of the gap and out-gapmodes. First of all we notice that our investigations of the gap solitons in the systems with 4, 6, 8, 10, 12, 16 partic-

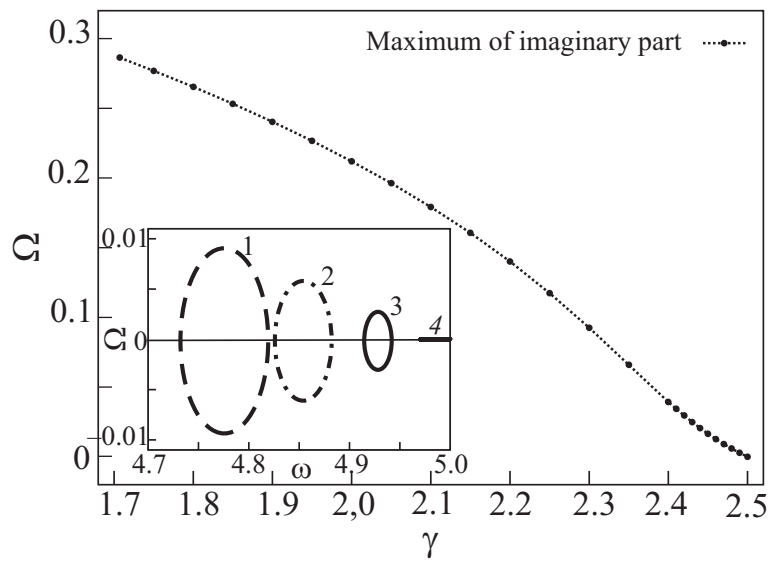

Fig. 3. The development of the Krein instability for the gap and out-gap breathers versus $\gamma: 2.47$ (1), 2.48 (2), 2.49 (3), $\geq 2.5$ (4). For $\gamma>2.35$ the oscillatory instability enters the gap regions $(\omega<4)$ and the resonance vanishes finally at $\gamma=2.5$ $(\omega=5)$. Close to the threshold of the stability the maximum value of the imaginary part of $\Omega$ shows a linear scaling in $\omega$ (and $\gamma$ ).

les have shown that the dependence of the gap and out-gap solution frequency on the norm changes qualitatively in the same manner when the number of particles grows or the parameter $\gamma$ grows. The following result is the most important: the dependence $\omega=\omega(N)$ does not embed into the lower zone of linear waves spectrum and remains inside the nonlinear gap of the spectrum, but it transforms essentially at the frequency of the lower boundary of the linear gap ( $\omega=4$ in Fig. 2). At this frequency value the transformation of the gap soliton into the out-gap one takes place. There are not analytical expressions for the gap and out-gap solitons, except the case $\gamma=2.5$ [9]. We studied the stability of these excitations numerically in the framework of Eqs. (3) with the use of numerical solutions for $\varphi_{n}$. The results are shown in Fig. 3. There exists the window of the Krein (oscillatory) instability of the gap and out-gap solitons analogues with nonzero imaginary part of parameter $\Omega$. But $\operatorname{Im} \Omega$ tends to zero while $\gamma \rightarrow 2.5$ (large depth of modulation). This justifies the expectation that the gap and out-gap solitons are stable in the large modulated systems. In the inset of Fig. 3 the transformation of the window of instability is presented: it lies inside the linear gap, the frequencies of the window and its width decrease with the growth of parameter $\gamma$.

\section{Conclusion}

The analogues of gap- and out-gap solitons have been studied in the quaternary fragment of discrete modulated nonlinear system of coupled oscillators. It has been demonstrated that transformation of such monochromatic 
soliton-like solutions and their stability depend essentially on the value of the modulating parameter $\gamma$. After two bifurcations at $\gamma=1.707$ and $\gamma=1.750$ the unified dependence of the soliton frequency $\omega$ on the norm $N$ for gap and out-gap solitons is formed. The gap soliton transforms into out-gap one at the lower boundary of the «linear gap» of the spectrum, while the dependence $\omega=\omega(N)$ for these excitations is situated above the lower boundary of «nonlinear gap». In the region $1.75<\gamma<2.5$ there exists the window of the oscillatory instability of the soliton solution, but for $\gamma>2.5$ the gap and out-gap solitons are stable for all the frequencies.

We would like to thank Magnus Johansson for valuable discussions. Financial support from the Royal Swedish Academy of Sciences is gratefully acknowledged.
1. D. Mills and J. Trullinger, Phys. Rev. B36, 947 (1987); W. Chen and D.L. Mills, Phys. Rev. Lett. 58, 160 (1987).

2. Yu.S. Kivshar and G.P. Agrawal, Optical Solitons, Academic Press, Amsterdam (2003).

3. J. Coste and J. Peyraud, Phys. Rev. B39, 13096 (1989).

4. A.M. Kosevich and A.S. Kovalev, Introduction in Nonlinear Physical Mechanics, Naukova dumka, Kiev (1989) (in Russian).

5. M.M. Bogdan, A.S. Kovalev, and E. Malyuta, Fiz. Nizk. Temp. 31, 807 (2005) [Low Temp. Phys. 31, 613 (2005)].

6. W. Wernsdorfer and R. Sessoli, Science 284, 133 (1999).

7. L. Kroon, Private Communication (2003).

8. J.E. Howard and R.S. MacKay, J. Math. Phys. 28, 10036 (1987).

9. M. Johansson, Private Communication (2005). 